\title{
Investigation of allele-specific expression of genes involved in adipogenesis and lipid metabolism suggests complex regulatory mechanisms of PPARGC1A expression in porcine fat tissues
}

\author{
Monika Stachowiak ${ }^{1 *}$, Izabela Szczerbal ${ }^{1}$ and Krzysztof Flisikowski ${ }^{2}$
}

\begin{abstract}
Background: The expression of genes involved in regulating adipogenesis and lipid metabolism may affect economically important fatness traits in pigs. Allele-specific expression (ASE) reflects imbalance between allelic transcript levels and can be used to identify underlying cis-regulatory elements. ASE has not yet been intensively studied in pigs. The aim of this investigation was to analyze the differential allelic expression of four genes, PPARA, PPARG, SREBF1, and PPARGCIA, which are involved in the regulation of fat deposition in porcine subcutaneous and visceral fat and longissimus dorsi muscle.

Results: Quantification of allelic proportions by pyrosequencing revealed that both alleles of PPARG and SREBF1 are expressed at similar levels. PPARGC1A showed the greatest ASE imbalance in fat deposits in Polish Large White (PLW), Polish Landrace and Pietrain pigs; and PPARA in PLW pigs. Significant deviations of mean PPARGC1A allelic transcript ratio between CDNA and genomic DNA were detected in all tissues, with the most pronounced difference $(p<0.001)$ in visceral fat of PLW pigs. To search for potential cis-regulatory elements affecting ASE in the PPARGC1A gene we analyzed the effects of four SNPs (rs337351686, rs340650517, rs336405906 and rs345224049) in the promoter region, but none were associated with ASE in the breeds studied. DNA methylation analysis revealed significant CpG methylation differences between samples showing balanced (allelic transcript ratio $\approx 1$ ) and imbalanced allelic expression for $\mathrm{CpG}$ site at the genomic position in chromosome 8 (SSC8): 18527678 in visceral fat $(p=0.017)$ and two CpG sites (SSC8:18525215, $p=0.030$; SSC8:18525237, $p=0.031$ ) in subcutaneous fat.

Conclusions: Our analysis of differential allelic expression suggests that PPARGC1A is subjected to cis-regulation in porcine fat tissues. Further studies are necessary to identify other regulatory elements localized outside the PPARGC1A proximal promoter region.
\end{abstract}

Keywords: Allele-specific expression, CpG methylation, Fat, Muscle, Pig, PPARA, PPARG, PPARGC1A, SREBF1

\footnotetext{
* Correspondence: dragan@up.poznan.pl

${ }^{1}$ Department of Genetics and Animal Breeding, Poznan University of Life

Sciences, Wolynska 33, 60-637 Poznan, Poland

Full list of author information is available at the end of the article
}

(c) The Author(s). 2018 Open Access This article is distributed under the terms of the Creative Commons Attribution 4.0 International License (http://creativecommons.org/licenses/by/4.0/), which permits unrestricted use, distribution, and reproduction in any medium, provided you give appropriate credit to the original author(s) and the source, provide a link to the Creative Commons license, and indicate if changes were made. The Creative Commons Public Domain Dedication waiver (http://creativecommons.org/publicdomain/zero/1.0/) applies to the data made available in this article, unless otherwise stated. 


\section{Background}

Fat deposition in pigs is a complex trait that is substantially influenced by multiple genetic factors, including regulatory mutations and epimutations affecting gene expression [1,2]. Gaining an understanding of factors involved in the regulation of lipid accumulation and fatty acid synthesis is important because excessive fatness has a negative effect on breeding efficiency. Moreover, consumers have become more interested in lean meat with high nutritional properties, and value the sensory attributes that are often influenced by fat content [3].

Allele-specific expression (ASE) contributes to the complexity of the transcriptome and reflects imbalance of expression between parental alleles. In contrast to random (e.g. X-chromosome inactivation), or non-random (imprinting) monoallelic expression where one allele is completely silenced, ASE is associated with more subtle differences in transcript level $[4,5]$. This can be a consequence of variations in cis-regulatory DNA regions involved in transcription efficiency or transcript stability, allele-specific DNA methylation, allele-specific histone modification or location of the chromosomal territory within the nucleus $[6,7]$. It is generally assumed that trans-regulatory elements do not contribute to ASE because both alleles are exposed to the same environmental factors. The frequency of ASE varies with species, tissue and individual physiological status, and analysis of its pattern may elucidate underlying regulatory mechanisms affecting many complex traits [8, 9]. About $20 \%$ of human genes are estimated to preferentially express one allele $[10,11]$, and recent high throughput RNA sequencing studies suggest it may be even more widespread [12]. For example, 52\% of genes exhibit ASE in pig brain [13], and $89 \%$ of bovine genes showed allelic imbalance in at least one of 18 tissues tested in a single individual [14]. Where the same allele is over-represented in unrelated heterozygous individuals (one-directional ASE), this suggests predominant regulation by cis-elements closely linked to the gene. In contrast, cases of ASE that show no such consistency (bi-directional ASE) suggest regulatory elements not in a strong linkage disequilibrium with the gene [15].

Allele-specific expression of genes encoding transcriptional regulators of adipogenesis and lipid metabolism may contribute to phenotypic variation of pig fatness traits because even subtle differences may affect their expression. Moreover, ASE can be used to explore the role of putative cis-regulatory factors. Here we investigated the allelic expression of PPARA (peroxisome proliferator activated receptor alpha), PPARG (peroxisome proliferator activated receptor gamma), PPARGC1A (PPARG coactivator 1 alpha) and SREBF1 (sterol regulatory element binding transcription factor 1 ) in subcutaneous and visceral fat deposits and in the skeletal longissimus dorsi ( $l$. dorsi) muscle of several pig breeds. We then investigated several potential cis-regulatory elements including genetic variants and DNA methylation in promoter regions for their possible role in tissue-specific ASE.

\section{Results}

\section{Genotyping and analysis of allele-specific expression}

We first identified animals heterozygous for an exonic reporter SNP (rSNP) in each gene to enable quantification of expression of each allele in transcript pools. One hundred forty-five pigs were genotyped (Additional file 1), and at least 10 heterozygotes found in Polish Large White (PLW) and Duroc for PPARA (rSNP: rs342258309, A > G); PLW, Polish Landrace (PL) and Duroc for PPARG (rSNP: rs319172675, A > G); PLW, PL, Duroc and Pietrain for PPARGC1A (rSNP: rs45430917, A > T); and PL, Duroc and Pietrain breed for SREBF1 (rSNP: rs712230598, C > T). Allelic transcript proportions were determined by pyrosequencing of cDNA reverse transcribed from RNA extracted from subcutaneous fat, visceral fat and $l$. dorsi muscle. Individual samples were classified as showing ASE when the allelic transcript ratio (percentage of one allele divided by the other) was $<0.667$ or $>1.5$, corresponding to allelic proportions greater than $40: 60$ or $60: 40$.

PPARG and SREBF1 showed no evidence of ASE in any sample (Additional file 2). Analysis of PPARA revealed ASE with preferential expression of allele A in two samples $(20 \%)$ of subcutaneous fat and one sample (10\%) of visceral fat from the PLW breed. The greatest allelic imbalance (allelic transcript ratio $=2.23$ ) was detected in the one sample in visceral fat (Additional file 2). Comparison of mean $\log _{10}$-transformed PPARG, SREBF1 and $P P A R A$ allelic transcript ratio between cDNA and gDNA in the breeds tested did not reveal statistically significant differences in the tissues studied (data not shown).

Analysis of PPARGC1A revealed markedly disproportionate allelic expression in individual samples of subcutaneous and visceral fat from PL, PLW and Pietrain pigs (Fig. 1). The nature of ASE was bi-directional, and the A/ $\mathrm{T}$ allelic transcript ratios varied between 0.57 and 3.52 in subcutaneous fat, and 0.41 and 3.22 in visceral fat (Fig. 2). ASE was more common in visceral fat of PLW (29\%) and PL pigs (27\%) than in subcutaneous fat (12 and 13\%, respectively). ASE was rare in Pietrain pigs (8\%) in both fat deposits. Analysis of mean $\log _{10}$-transformed allelic transcript ratios between cDNA and genomic DNA revealed significant deviations in subcutaneous fat of PLW, PL and Duroc pigs, in visceral fat of all breeds and in $l$. dorsi muscle of PL and Duroc pigs (Table 1). The most significant differences $(p<0.001)$ were observed in visceral fat of PLW pigs. 


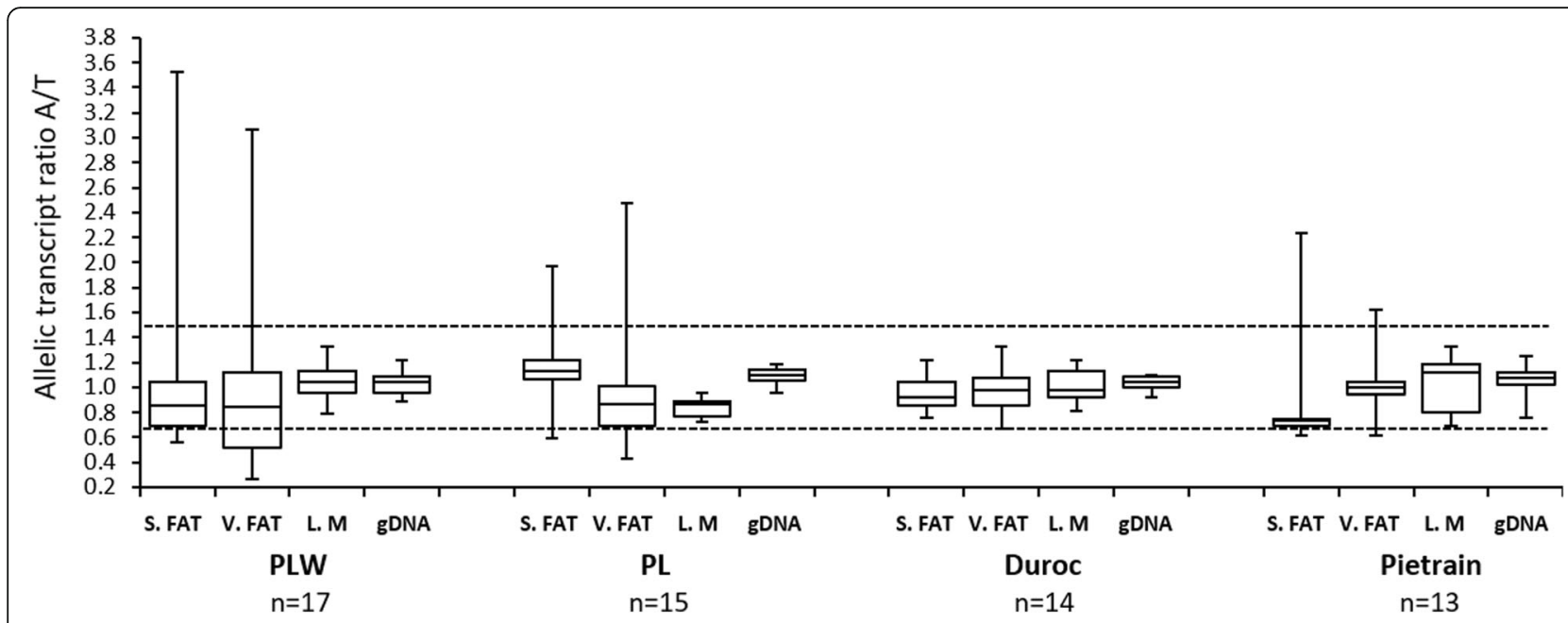

Fig. 1 Distribution of allelic transcript ratios for PPARGC1A in tissues and genomic DNA of analyzed breeds. Each boxplot shows the first quartile, median, third quartile and the whiskers show the minimum and maximum allelic transcript ratio values. S. FAT - subcutaneous fat, V. FAT visceral fat, L. M. - longissimus dorsi muscle, gDNA - genomic DNA

These data led us investigate potential cis-regulatory factors that may affect PPARGC1A expression in porcine tissues.

\section{Potential regulatory variants in the PPARGC1A 5'-flanking region}

A $\sim 1 \mathrm{~kb}$ region upstream of the translation initiation site, including the entire $5{ }^{\prime} \mathrm{UTR}$ ( $5^{\prime}$-untranslated region) and the proximal promoter was sequenced to search for DNA polymorphisms. Four SNPs were identified in the promoter: rs331429264 (c.-393G > C), rs337351686 (c.-530G > A), rs318575008 (c.-531C > G) and rs340650517 (c.-644G > A), but none in the $5{ }^{\prime} \mathrm{UTR}$. We also genotyped the rs336405906 (c.-2885G > T) and rs345224049 (c.-2894G >

A) SNPs in the distal promoter region to verify their effects on PPARGC1A mRNA expression previously reported by Kim et al. [16]. In silico analysis predicted that three of the SNPs disrupt putative consensus sites for transcription factors expressed in adipose tissue and/or skeletal muscle (Additional file 3). Of these, the ATF2 binding sequence was disturbed by rs331429264, that of STAT5A and STAT5B by rs337351686, and KLF4 and TP53 by rs340650517. These transcription factors are known regulators of adipogenesis, adipocyte function and have been

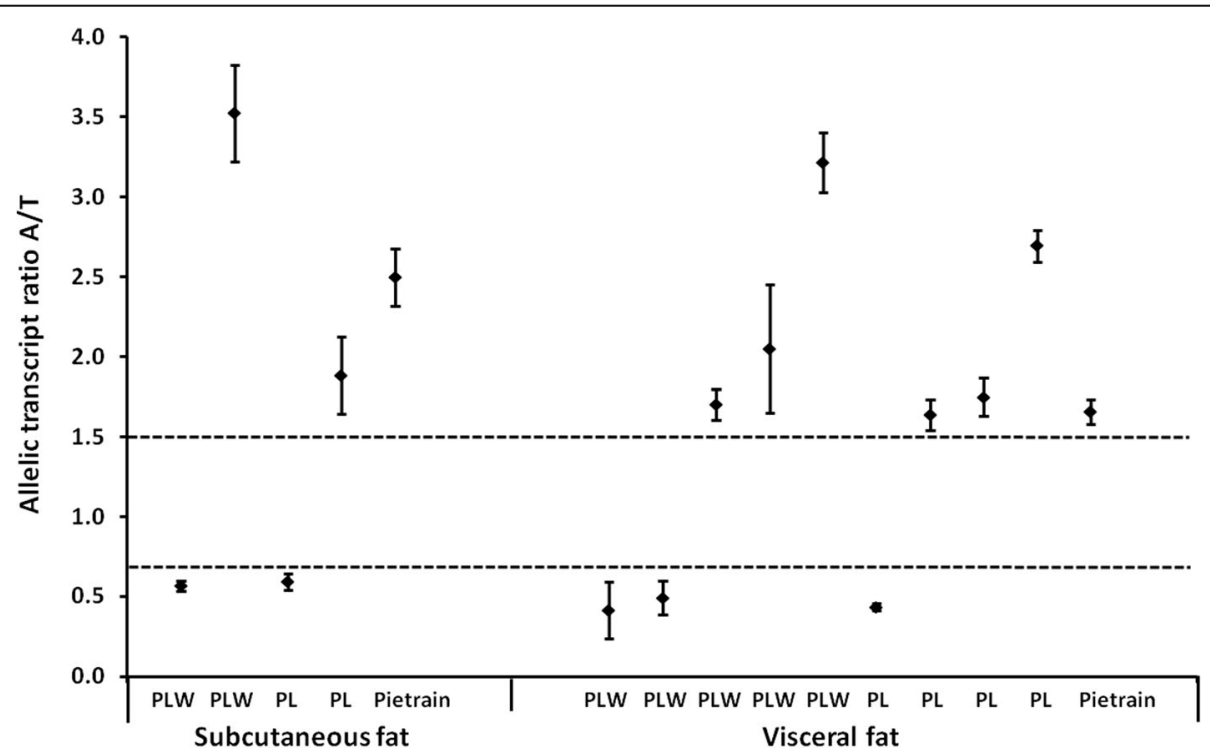

Fig. 2 Allelic transcript ratios (mean of two measurements) \pm SD of individual samples showing ASE of PPARGC1A in subcutaneous and visceral fat. Threshold allelic transcript ratio values (0.667 and 1.5) are marked with a dashed line 
Table 1 Mean $\log _{10}$-transformed PPARGC1A allelic transcript ratios in CDNA derived from subcutaneous fat, visceral fat and $I$. dorsi muscle, and genomic DNA (gDNA). Data were calculated after neutralizing bi-directional nature of PPARGC1A allelic expression

\begin{tabular}{lllll}
\hline Breed $^{\mathrm{a}}$ & Subcutaneous fat $^{\mathrm{b}}$ & Visceral fat $^{\mathrm{b}}$ & L. dorsi muscle $^{\mathrm{b}}$ & $\mathrm{gDNA}$ \\
\hline PLW & $0.149^{* *}$ & $0.176^{* * *}$ & 0.046 & 0.032 \\
PL & $0.072^{*}$ & $0.130^{* *}$ & $0.054^{* *}$ & 0.021 \\
Duroc & $0.052^{*}$ & $0.070^{* *}$ & $0.047^{* *}$ & 0.022 \\
Pietrain & 0.077 & $0.094^{*}$ & 0.041 & 0.040 \\
\hline
\end{tabular}

${ }^{a} P L W$ Polish Large White, PL Polish Landrace

${ }^{\mathrm{b}}$ Significant differences between CDNA and gDNA are shown at $p<0.05\left(^{*}\right)$, $p<0.01\left(^{* *}\right)$ and $p<0.001\left(^{* * *}\right)$

implicated in human obesity and fatness traits in animals (Additional file 3). ATF2 and TP53 have been reported to regulate PPARGC1A promoter activity $[17,18]$. To examine the association between promoter SNPs and PPARGC1A mRNA expression, we compared allelic transcript ratios derived from animals carrying different genotypes. This approach reduces possible confounding effects of trans-regulatory and environmental factors on mRNA expression because transcript abundance is compared within the same sample, not between samples [19]. Due to sufficient genotype distributions (Additional file 4), we could apply this method to test regulatory effects of rs340650517, rs337351686 and rs336405906 in PLW, and rs345224049 in PL pigs. We found that rs340650517, rs337351686 and rs336405906 SNPs segregated in PLW pigs as two haplotypes $[\mathrm{G} ; \mathrm{G} ; \mathrm{G}]$ and $[\mathrm{A} ; \mathrm{A} ; \mathrm{T}]$ and were thus analyzed together. The comparison of mean $\log _{10}$-transformed allelic transcript ratios between $[\mathrm{G} ; \mathrm{G} ; \mathrm{G}] /[\mathrm{G} ; \mathrm{G} ; \mathrm{G}]$ and $[\mathrm{G} ; \mathrm{G} ; \mathrm{G}] /[\mathrm{A} ; \mathrm{A} ; \mathrm{T}]$ diplotype groups revealed no significant effect on allelic transcript ratio in subcutaneous $(p=0.25)$ or visceral fat $(p$ $=0.83)$, or $l$. dorsi muscle $(p=0.25)$. Similarly, rs345224049 SNP did not significantly affect the allelic transcript ratio in subcutaneous $(p=0.22)$ or visceral fat deposits $(p=0.37)$, or $l$. dorsi muscle $(p=0.08)$. We thus conclude that the observed ASE of PPARGC1A did not result from regulatory effects of rs337351686, rs340650517, rs336405906 and rs345224049 SNPs in the analyzed samples.

\section{CpG methylation analysis in the PPARGC1A region}

A search for epigenetic regulatory elements that may affect expression of PPARGC1A transcripts via CpG methylation was performed at three $\mathrm{CpG}$ islands (CGi) selected based on porcine genome data (Sscrofa11.1) for chromosome 8 (SSC8). CGi1 and CGi2 are located in the $5^{\prime}$-flanking region (genomic positions SSC8:18527230-18528335 and 18524520-18526314, respectively) and CGi3 in exon 6 of PPARGC1A (SSC8:17866933-17867385). We compared 5-methylcytosine (5-mC) levels (\%) in subcutaneous and visceral fat tissues between samples displaying ASE (allelic transcript ratio exceeding $0.667-1.5$ range) and control samples with similar expression of both alleles (mean allelic transcript ratio \pm SD was $0.98 \pm 0.09$ in subcutaneous fat and $1.02 \pm 0.11$ in visceral fat). The mean $\mathrm{CpG}$ methylation level at CGi1 and CGi2 was found to be low (2-6\% and 1$4 \%$, respectively; Fig. 3 ) in the samples analyzed. In subcutaneous fat, DNA methylation was significantly higher in ASE samples for two CpG sites within CGi2 at genomic positions in chromosome 8:18525215 $(p=0.030)$ and 8:18525237 $(p=0.031)$. In visceral fat, the CpG site at the position 8:18527678 was significantly higher methylated in ASE samples than in a control group $(p=0.017)$ (Fig. 3). For intragenic CGi3, the mean DNA methylation was high $(67-97 \%)$ in both fat deposits, but did not differ statistically between ASE and control samples (Additional file 5).

\section{Discussion}

Allelic imbalance in genes involved in regulating porcine lipid metabolism and fat tissue physiology has not so far been widely investigated, and there are only a few reports that relate transcriptome based allele specific expression to lipid metabolism or fatness traits. In a study where $l$. dorsi muscles were analyzed during prenatal development, a total of 11,300 variants showed allelic imbalance [20]. Of these, 3 SNPs in SCD, NR3C1 and PGM1 were associated with porcine fatness and growth traits. Esteve-Codina et al. [21] investigated testicular transcriptome in two pigs with extremely divergent phenotypes including fatness traits. The lipid metabolism category was overrepresented by differentially expressed genes but ASE was detected for only $4 \%$ of transcripts. On the other hand, Schachtschneider et al. [22] reported that of eight porcine tissues tested, fat showed the greatest number of ASE genes (225) and muscle the least (135). These studies analyzed single animals and the ASE results were not validated by an alternative method. RNA-seq technology can detect genes with imbalanced allelic expression but may produce false positive ASE hits [23]. Allelic expression imbalance has also been analyzed for several genes thought to affect pig meat quality and fatness traits. Deviations between allelic transcript levels were detected for ADRB2 in $l$. dorsi muscle [24], SERPINA6 and $A P O A 2$ in liver $[25,26]$ but not for $A D I P O Q$ in skeletal muscles and backfat [27]. Our study analyzed ASE of four functional candidate genes encoding transcription factors (PPARA, PPARG, SREBF1) and a coactivator of multiple transcription factors (PPARGC1A) using pyrosequencing as an accurate and sensitive means of quantifying allelic transcript proportions [28].

We demonstrated that both alleles of PPARG and SREBF1 are expressed at similar levels in subcutaneous and visceral fat and $l$. dorsi muscle in 30 unrelated animals representing three commercial pig breeds. Interestingly, recent studies of human PPARG revealed allele-specific expression in adipose tissue and led to the identification 

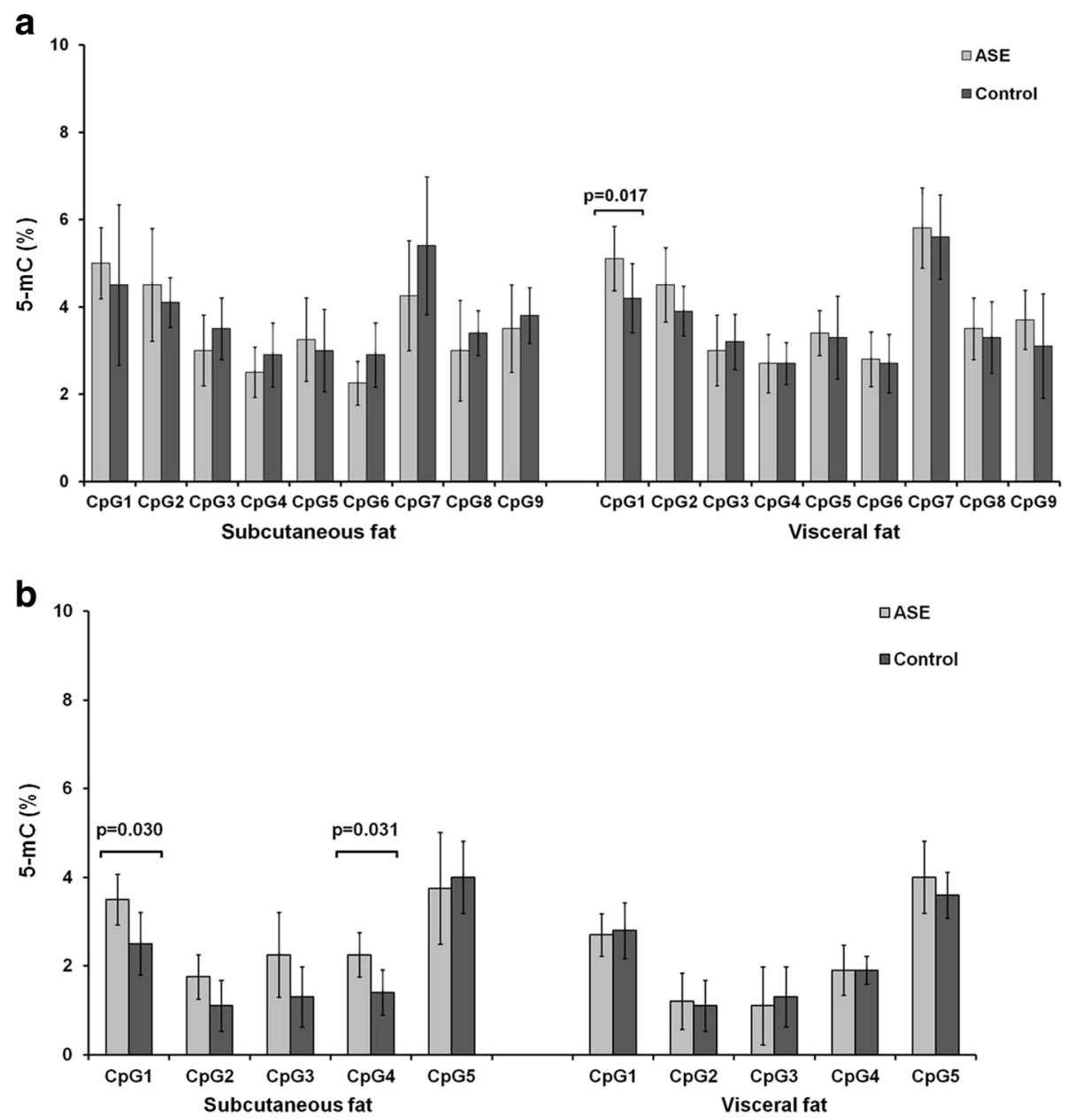

Fig. 3 Mean percentage of 5-methylcytosine (5-mC) $\pm S D$ within CpG islands located in 5'-flanking region of PPARGC1A in fat deposits. The particular cytosines in each fragment analyzed: CGi1 (a) and CGi2 (b), are indicated as CpG1, CpG2, etc. $P$ value is shown for cytosines that differed significantly in methylation level between ASE samples ( $n=5$ for subcutaneous fat and $n=10$ for visceral fat) and control groups ( $n=10$ for subcutaneous and $n=10$ for visceral fat) with similar expression of both alleles

of a regulatory variant rs4684847 that is associated with risk of type 2 diabetes $[29,30]$.

Although we detected single PLW individuals showing ASE of PPARA in subcutaneous and visceral fat, the mean allelic transcript ratios between cDNA and genomic DNA did not differ significantly, probably due to the small number of individual samples analyzed. We did not detect any other exonic SNP in PPARA that could serve as a reporter SNP in our populations that would have enabled allele discrimination in a larger number of heterozygotes, a common limiting factor in such studies. Because PPARA is an interesting candidate gene associated with numerous porcine fatness traits [31, 32], further investigation of possible cis-regulatory elements affecting its expression is recommended.

Differential allelic expression occurred most frequently for PPARGC1A, and bi-directional ASE (A or T allele overrepresented) was detected in fat deposits of PL, PLW and Pietrain pigs. Of the tissues analyzed, we found the greatest number of samples showing allelic imbalance in visceral fat. Tissue and site-specific regulation and function of PPARGC1A have been previously highlighted by the finding that its expression level depends on the anatomical location (3rd-4th rib or near the 4th lumbar vertebra) within the porcine $l$. dorsi muscle [33].

The PPARGC1A gene encodes a versatile coactivator of many nuclear receptor families, including PPARs (peroxisome proliferator activated receptors) and is involved in stimulation of mitochondrial biogenesis, regulation of glucose, fatty-acid metabolism and muscle fiber type formation [34]. PPARGC1A is thus a promising candidate gene for fatness and meat quality traits in livestock, and there have been several reports of associations of its polymorphism or expression with relevant pig production traits [33, 
35-38]. Ours is the first study to show ASE of PPARGC1A in mammalian tissues.

ASE may be a useful means of identifying and dissecting the genetic or epigenetic factors responsible for differences in transcript expression. A search for potential cis-acting regulatory variants using quantification of allelic proportions has successfully identified a variant affecting expression of human IL13 [19]. Based on association between environment and ASE some risk factors for complex diseases were identified in humans. For example, increased allelic imbalance of VNN1 in whole blood was associated with elevated BMI [39]. In another study, allele-specific expression of several genes in islets (ANPEP, CAMK2B, HMG20A, KCNJ11, NOTCH2, SLC3OA8 and WFS1) showed that even subtle changes of gene dosage may have significant consequences for development of type 2 diabetes [40]. These results indicate that analysis of ASE is useful to understand pathophysiology of human diseases. It can be anticipated that application of this approach in livestock studies will allow identify molecular background of complex traits, including fatness.

Here, we attempted to decipher potential regulatory elements in PPARGC1A but the promoter SNPs identified (rs337351686, rs340650517, rs336405906 and rs345224049) did not affect allelic transcript ratios. An earlier study reported that genes displaying bi-directional ASE show greater variability in methylation of promoter CpG sites than genes with one-directional ASE [41]. Thus, we analyzed methylation at $\mathrm{CpG}$ pairs in the $5^{\prime}$-flanking region as well as in exon 6 of PPARGC1A. The role of gene body methylation as an epigenetic mark is not clear, but it is known that exons are more highly methylated than intergenic regions, and the gene body methylation level is positively correlated with gene expression [22, 42]. We compared methylation status between samples showing similar and imbalanced expression of two PPARGC1A alleles for two CpG islands in the $5^{\prime}$-flanking region. We found subtle but statistically significant differences in methylation level at two CpG sites in subcutaneous fat and one CpG in visceral fat. Although small, these differences may potentially contribute to variation in allelic expression because they may indicate expression changes in particular cell types. The importance of $\mathrm{CpG}$ methylation within the PPARGC1A promoter to adiposity has been highlighted by the finding that its methylation status in early childhood can predict body fat percent in older children [43].

The bi-directional nature of ASE indicates that regulatory elements affecting PPARGC1A are not in linkage disequilibrium with the exonic rSNP (rs45430917) used to quantify allele proportions. Our results showed no evidence that promoter SNPs that were commonly distributed in PLW and PL heterozygotes for rSNP affected PPARGC1A allelic expression. We suggest that the PPARGC1A may be subject to complex regulation, probably by long-range regulatory factors such as genetic variants or cis-regulatory chromatin modifications but CpG methylation may be to some extent involved. Future studies are necessary to identify functional cis-acting elements that affect PPARGC1A allelic expression in pig tissues.

\section{Conclusions}

Ours is the first study of allele-specific expression of candidate genes involved in regulation of adipogenesis and lipid metabolism in subcutaneous fat, visceral fat and $l$. dorsi muscle of several pig breeds. PPARG and $S R E B F 1$ allele expression was balanced. Some imbalance of allelic transcripts was found for PPARA, but the greatest prevalence of ASE was detected for PPARGC1A in visceral fat of Polish Large White and Polish Landrace pigs. The bi-directional character of ASE of PPARGC1A shows that underlying cis-regulatory elements are not in linkage disequilibrium with the SNP used to measure allelic proportions. We detected small differences of DNA methylation levels within $\mathrm{CpG}$ islands that can be associated with PPARGC1A allelic expression in subcutaneous and visceral fat. Further studies are necessary to identify other regulatory elements localized outside the proximal promoter region of PPARGC1A.

\section{Methods}

\section{Animals and tissue collection}

A total of 145 female pigs representing Polish Large White (PLW; $n=51$ ), Polish Landrace (PL; $n=35$ ), Duroc $(n=38)$ and Pietrain $(n=21)$ breeds were analyzed. Gilts were kept under identical environmental conditions, fed ad libitum with the same commercial mix fodder, slaughtered at $100 \mathrm{~kg}(\mathrm{SD}=1.8)$ weight and dissected at the local Pig Testing Station (Pawlowice, Poland). Peripheral blood, longissimus dorsi (l. dorsi) muscle, subcutaneous and visceral fat tissues were collected. Tissue samples were snap frozen in liquid nitrogen and stored at $-80^{\circ} \mathrm{C}$.

\section{Genotyping}

Genomic DNA for genotyping was isolated from peripheral blood of 145 pigs using Blood Mini kit (A\&A Biotechnology). PCR reactions were performed using primers overlapping known exonic SNPs that were used as reporter SNPs (rSNP) to quantify allele-specific expression: rs342258309 (A > G) in PPARA, rs319172675 $(\mathrm{A}>\mathrm{G})$ in PPARG, rs45430917 (A > T) in PPARGC1A, and rs712230598 $(\mathrm{C}>\mathrm{T})$ in SREBF1. For SREBF1, genotypes of some pigs were retrieved from our previous study [44]. Sequences of PCR primers are shown in Additional file 6. Prior to Sanger sequencing amplicons 
were purified using Exonuclease I (Thermo Scientific) and Alkaline Phosphatase (Thermo Scientific) and sequencing PCR performed using BigDye Terminator v.3 Cycle Sequencing kit (Thermo Scientific). Sequencing products were filtered on Sephadex G-50 (SigmaAldrich) and separated by capillary electrophoresis on 3130 Genetic Analyzer (Applied Biosystems).

\section{Quantification of allele proportions}

Quantitative analysis was performed only for breeds where at least 10 heterozygotes were found for each rSNP. Total RNA was extracted from tissue samples using Direct-zol RNA MiniPrep kit (Zymo Research) and TriPure Isolation Reagent (Roche) according to manufacturer's protocol. Prior to cDNA synthesis, $1 \mu \mathrm{g}$ RNA was digested with DNase I (Sigma-Aldrich) to remove contaminating genomic DNA. One-strand cDNA synthesis was performed using a Transcriptor High Fidelity cDNA Synthesis Kit (Roche). All cDNA samples were tested for the presence of contaminating genomic DNA by a PCR reaction with primers specific for genomic DNA and cDNA. The cDNA samples were then used to amplify fragments encompassing analyzed SNPs that were used for allele quantification by pyrosequencing. Assays were designed using PyroMark Assay Design 2.0 software (Qiagen). Sequences of primers used for PCR amplifications and pyrosequencing are shown in Additional file 6. Pyrosequencing reactions were performed using PyroMark Q48 Advanced Reagents (Qiagen) and analyzed on Pyromark Q48 Autoprep system (Qiagen). Allelic proportions were first quantified as a percentage of incorporated nucleotides for the tested rSNP using Pyromark Q48 Autoprep 2.4.2 software (Qiagen) and allelic ratios were then calculated by dividing the percentage of one allele by the other. PCR products obtained from genomic DNA (gDNA) and amplified with the same primer pairs as cDNA samples were also pyrosequenced. In gDNA of heterozygotes, an equimolar ratio of both allelic transcripts is expected. Any bias resulting from variations in nucleotide incorporation during pyrosequencing reaction was normalized by dividing the allelic ratio of cDNA and gDNA samples by a mean allelic ratio derived from gDNA for each gene tested [45, 46]. Individual samples were defined as imbalanced when the allelic proportion (mean of two measurements) exceeded a 60:40 threshold [46], i.e. allelic transcript ratios were $>1.5$ for one-directional and $<0.667$ or $>1.5$ for bi-directional ASE. The deviation of mean allelic expression between cDNA and gDNA for each breed was tested by two-tailed $t$ test with unequal variances using $\log _{10}$ -transformed allelic transcript ratios [19, 45]. For PPARGC1A, the bi-directional character of ASE was neutralized by dividing the higher percentage by the lower as previously described [46].

\section{Promoter sequence analysis}

To search for polymorphic variants potentially associated with ASE, Sanger sequencing of two amplicons encompassing $1010 \mathrm{bp}$ of the proximal promoter and $5{ }^{\prime}$-untranslated region ( $\left.5^{\prime} \mathrm{UTR}\right)$ of PPARGC1A was performed as described above. In addition, rs336405906 (c.-2885G > T) and rs345224049 (c.-2894G > A) SNPs were genotyped as putatively associated with PPARGC1A expression according to Kim et al. [16]. Sequences of PCR primers are shown in Additional file 6. MatInspector software (Genomatrix) was used for in silico analysis of putative transcription factor binding sites. Haplotypes were predicted using Haploview (Broad Institute). Association between promoter SNP and allelic transcript expression was performed using a two-tailed $t$ test with unequal variances of $\log _{10}$-transformed and phase-corrected allelic transcript ratios of heterozygous versus homozygous individuals as described by Forton et al. [19].

\section{CpG methylation analysis}

Two CGi in 5'-flanking region (CGi1 SSC8:1852723018528335; CGi2 SSC8:18524520-18526314) based on NCBI database and in exon 6 of PPARGC1A (CGi3 SSC8:17866933-17867385) based on ENSEMBL database, were selected and primers for CpG methylation analysis (Additional file 6) were designed using PyroMark Assay Design 2.0 software (Qiagen). DNA was purified from subcutaneous and visceral fat by phenol:chloroform:isoamyl alcohol (25:24:1, Sigma-Aldrich) extraction. Methylated and unmethylated controls were prepared with $\mathrm{CpG}$ Methyltransferase (Thermo Scientific) and REPLI-g Mini Kit (Qiagen), respectively. Five hundred nanograms DNA was bisulfite-converted using a EZ DNA Methylation -Gold Kit (Zymo Research). PCR reactions were performed on bisulfite-converted DNA using a Pyromark PCR Kit (Qiagen) according to manufactuer's recommendations. Methylation analysis was carried out by pyrosequencing using Pyromark Q48 Advanced CpG reagents (Qiagen) and analyzed on Pyromark Q48 Autoprep system (Qiagen). CpG methylation level (\%) was compared with Student $t$ test between ASE samples $(n=5$ for subcutaneous fat and $n=10$ for visceral fat) and control samples $(n=10$ for subcutaneous and $n=10$ for visceral fat) with similar expression of both alleles.

\section{Additional files}

Additional file 1: Genotype frequencies of rSNPs in PPARA, PPARG, PPARGC1A and SREBF genes in tested pig breeds. (DOC $42 \mathrm{~kb}$ )

Additional file 2: Distribution of allelic transcript ratios for a) $P P A R G$; b) SREBF1; c) PPARA in tissues and genomic DNA of analyzed breeds. Each boxplot shows the first quartile, median, third quartile and the whiskers show the minimum and maximum allelic transcript ratio values. S. FAT subcutaneous fat, V. FAT - visceral fat, L. M. - longissimus dorsi muscle, gDNA - genomic DNA. (TIF $87 \mathrm{~kb}$ ) 
Additional file 3: SNPs affecting transcription factor (TF) binding to PPARGCIA promoter, TF tissue expression and consensus sequences. (DOC $41 \mathrm{~kb}$ )

Additional file 4: Genotype frequencies for SNPs in PPARGC1A 5'flanking sequence in heterozygous samples for exonic reporter SNP rs45430917. (DOC $32 \mathrm{~kb}$ )

Additional file 5: Mean percentage of 5-methylcytosine (5-mC) \pm SD within CGi3, localized in exon 6 of PPARGC1A in fat deposits of ASE samples and control groups. The particular cytosines in each fragment analyzed are indicated as CpG1, CpG2, etc. ASE groups included $n=5$ samples for subcutaneous fat and $n=10$ samples for visceral fat. Control groups with similar expression of both alleles included $n=10$ samples for subcutaneous and $n=10$ samples for visceral fat. (TIF $1950 \mathrm{~kb}$ )

Additional file 6: $P C R$ primers used for genotyping, quantification of allelic transcript proportions and CpG methylation analysis. (DOC $46 \mathrm{~kb}$ )

\section{Abbreviations \\ 5-mC: 5-methylcytosine; ASE: Allele-specific expression; BMI: Body mass index; CGi: CpG island; I. dorsi: Longissimus dorsi; PL: Polish Landrace; PLW: Polish Large White; PPARA: Peroxisome proliferator activated receptor alpha; PPARG: Peroxisome proliferator activated receptor gamma; PPARGC1A: PPARG coactivator 1 alpha; rSNP: Reporter SNP; SD: Standard deviation; SNP: Single nucleotide polymorphism; SREBF1: Sterol regulatory element binding transcription factor; SSC8: SUS scrofa chromosome 8; UTR: Untranslated region}

\section{Acknowledgements}

We thank Alexander Kind of the Chair of Livestock Biotechnology, Technical University of Munich, Germany for English editing.

\section{Funding}

This study was supported by the National Science Center in Poland, grant no. 2012/07/E/NZ9/02573 and the Young Researcher Program of the Faculty of Veterinary Medicine and Animal Science Poznan University of Life Sciences, Poland financed by the Polish Ministry of Science and Higher Education, grant no. 508.534.00.0. The funding body had no role in the design of the study, collection of research materials, analysis and interpretation of the data and in writing the manuscript.

\section{Availability of data and materials}

The datasets supporting the conclusions of this article are included within the article and its additional files.

\section{Authors' contributions}

MS and IS designed the study. MS and KF conducted the experiments. MS analyzed the data and wrote the paper. All authors read, revised and approved the final manuscript.

\section{Ethics approval and consent to participate}

All animal procedures performed for the purpose of the study were approved by the Local Ethical Commission on Experiments on Animals at the Poznan University of Life Sciences, Poznan, Poland (no. 57/2012).

\section{Consent for publication}

Not Applicable

\section{Competing interests}

The authors declare that they have no competing interests.

\section{Publisher's Note}

Springer Nature remains neutral with regard to jurisdictional claims in published maps and institutional affiliations.

\section{Author details}

'Department of Genetics and Animal Breeding, Poznan University of Life Sciences, Wolynska 33, 60-637 Poznan, Poland. ${ }^{2}$ Chair of Livestock Biotechnology, Technical University of Munich, Liesel-Beckmannstr. 1, 85354 Freising, Germany.
Received: 5 July 2018 Accepted: 19 November 2018

Published online: 29 November 2018

\section{References}

1. Switonski M, Stachowiak M, Cieslak J, Bartz M, Grzes M. Genetics of fat tissue accumulation in pigs: a comparative approach. J Appl Genet. 2010;51:153-68.

2. Stachowiak M, Szczerbal I, Switonski M. Genetics of adiposity in large animal models for human obesity-studies on pigs and dogs. Prog Mol Biol Transl Sci. 2016;140:233-70.

3. Pena RN, Ros-Freixedes R, Tor M, Estany J. Genetic marker discovery in complex traits: a field example on fat content and composition in pigs. Int J Mol Sci. 2016;17:2100.

4. Chess A. Random and non-random monoallelic expression. Neuropsychopharmacology. 2013;38:55-61.

5. Bell CG, Beck S. Advances in the identification and analysis of allele-specific expression. Genome Med. 2009;1:56.

6. Gaur U, Li K, Mei S, Liu G. Research progress in allele-specific expression and its regulatory mechanisms. J Appl Genet. 2013;54:271-83.

7. Kociucka B, Cieslak J, Szczerbal I. Three-dimensional arrangement of genes involved in lipid metabolism in nuclei of porcine adipocytes and fibroblasts in relation to their transcription level. Cytogenet Genome Res. 2012;136:295-302.

8. Crowley JJ, Zhabotynsky V, Sun W, Huang S, Pakatci IK, Kim Y, et al. Analyses of allele-specific gene expression in highly divergent mouse crosses identifies pervasive allelic imbalance. Nat Genet. 2015;47:353-60.

9. Wu H, Gaur U, Mekchay S, Peng X, Li L, Sun H, et al. Genome-wide identification of allele-specific expression in response to Streptococcus suis 2 infection in two differentially susceptible pig breeds. J Appl Genet. 2015;56:481-91

10. Serre D, Gurd S, Ge B, Sladek R, Sinnett D, Harmsen E, et al. Differential allelic expression in the human genome: a robust approach to identify genetic and epigenetic cis-acting mechanisms regulating gene expression. PLoS Genet. 2008:4:e1000006.

11. Dimas $A S$, Stranger BE, Beazley $C$, Finn $R D$, Ingle $C E$, Forrest $M S$, et al. Modifier effects between regulatory and protein-coding variation. PLoS Genet. 2008;4:e1000244.

12. Xu H, Pausch $H$, Venhoranta $H$, Rutkowska K, Wurmser $C$, Rieblinger $B$, et al. Maternal placenta modulates a deleterious fetal mutation. Biol Reprod. 2017:97:249-57.

13. Oczkowicz M, Szmatola T, Piorkowska K, Ropka-Molik K. Variant calling from RNA-seq data of the brain transcriptome of pigs and its application for allele-specific expression and imprinting analysis. Gene. 2018;641:367-75.

14. Chamberlain AJ, Vander Jagt CJ, Hayes BJ, Khansefid M, Marett LC, Millen $C A$, et al. Extensive variation between tissues in allele specific expression in an outbred mammal. BMC Genomics. 2015;16:993.

15. Pant PV, Tao H, Beilharz EJ, Ballinger DG, Cox DR, Frazer KA. Analysis of allelic differential expression in human white blood cells. Genome Res. 2006;16:331-9.

16. Kim JM, Lim KS, Lee EA, Lee KT, Kim TH, Ryu YC, et al. Polymorphisms of the 5 ' regulatory region of the porcine PPARGC1A gene and the effects on muscle fiber characteristics and meat quality. Mol Biol Rep. 2012;39:3933-42.

17. Aquilano K, Baldelli S, Pagliei B, Cannata SM, Rotilio G, Ciriolo MR. p53 orchestrates the PGC-1alpha-mediated antioxidant response upon mild redox and metabolic imbalance. Antioxid Redox Signal. 2013;18:386-99.

18. Karamitri A, Shore AM, Docherty K, Speakman JR, Lomax MA. Combinatorial transcription factor regulation of the cyclic AMP-response element on the Pgc-1alpha promoter in white 3T3-L1 and brown HIB-1B preadipocytes. J Biol Chem. 2009;284:20738-52.

19. Forton JT, Udalova IA, Campino S, Rockett KA, Hull J, Kwiatkowski DP. Localization of a long-range cis-regulatory element of IL13 by allelic transcript ratio mapping. Genome Res. 2007;17:82-7.

20. Yang $Y$, Tang Z, Fan $X, X u K$, Mu Y, Zhou R, et al. Transcriptome analysis revealed chimeric RNAs, single nucleotide polymorphisms and allele-specific expression in porcine prenatal skeletal muscle. Sci Rep. 2016;6:29039.

21. Esteve-Codina A, Kofler R, Palmieri N, Bussotti G, Notredame C, Perez-Enciso M. Exploring the gonad transcriptome of two extreme male pigs with RNAseq. BMC Genomics. 2011;12:552.

22. Schachtschneider KM, Madsen O, Park C, Rund LA, Groenen MA, Schook LB. Adult porcine genome-wide DNA methylation patterns support pigs as a biomedical model. BMC Genomics. 2015;16:743. 
23. Fresard L, Leroux S, Servin B, Gourichon D, Dehais P, Cristobal MS, et al. Transcriptome-wide investigation of genomic imprinting in chicken. Nucleic Acids Res. 2014;42:3768-82.

24. Murani E, Ponsuksili S, Srikanchai T, Maak S, Wimmers K. Expression of the porcine adrenergic receptor beta 2 gene in longissimus dorsi muscle is affected by cis-regulatory DNA variation. Anim Genet. 2009:40:80-9.

25. Gorres A, Ponsuksili S, Wimmers K, Murani E. Analysis of non-synonymous SNPS of the porcine SERPINA6 gene as potential causal variants for a QTL affecting plasma cortisol levels on SSC7. Anim Genet. 2015;46:239-46.

26. Ballester M, Revilla M, Puig-Oliveras A, Marchesi JA, Castello A, Corominas J, et al. Analysis of the porcine APOA2 gene expression in liver, polymorphism identification and association with fatty acid composition traits. Anim Genet. 2016;47:552-9.

27. Castello A, Quintanilla R, Melo C, Gallardo D, Zidi A, Manunza A, et al. Associations between pig adiponectin (ADIPOQ) genotype and serum lipid levels are modulated by age-specific modifiers. J Anim Sci. 2014;92:5367-73.

28. Wang $\mathrm{H}$, Elbein SC. Detection of allelic imbalance in gene expression using pyrosequencing. Methods Mol Biol. 2007;373:157-76.

29. Claussnitzer M, Dankel SN, Klocke B, Grallert H, Glunk V, Berulava T, et al. Leveraging cross-species transcription factor binding site patterns: from diabetes risk loci to disease mechanisms. Cell. 2014;156:343-58.

30. Lee H, Qian K, von Toerne C, Hoerburger L, Claussnitzer M, Hoffmann $C$, et al. Allele-specific quantitative proteomics unravels molecular mechanisms modulated by cis-regulatory PPARG locus variation. Nucleic Acids Res. 2017:45:3266-79.

31. Puig-Oliveras A, Revilla M, Castello A, Fernandez Al, Folch JM, Ballester M Expression-based GWAS identifies variants, gene interactions and key regulators affecting intramuscular fatty acid content and composition in porcine meat. Sci Rep. 2016;6:31803.

32. Stachowiak M, Szydlowski M, Flisikowski K, Flisikowska T, Bartz M, Schnieke $A$, et al. Polymorphism in 3' untranslated region of the pig PPARA gene influences its transcript level and is associated with adipose tissue accumulation. J Anim Sci. 2014;92:2363-71.

33. Erkens T, Vandesompele J, Van Zeveren A, Peelman LJ. Correlation between porcine PPARGC1A mRNA expression and its downstream target genes in backfat and longissimus dorsi muscle. J Appl Genet. 2009:50:361-9.

34. Liu C, Lin JD. PGC-1 coactivators in the control of energy metabolism. Acta Biochim Biophys Sin Shanghai. 2011;43:248-57.

35. Gandolfi G, Cinar MU, Ponsuksili S, Wimmers K, Tesfaye D, Looft C, et al. Association of PPARGC1A and CAPNS1 gene polymorphisms and expression with meat quality traits in pigs. Meat Sci. 2011:89:478-85.

36. Stachowiak M, Szydlowski M, Cieslak J, Switonski M. SNPs in the porcine PPARGC1a gene: interbreed differences and their phenotypic effects. Cell Mol Biol Lett. 2007;12:231-9.

37. Li Q, Wang Z, Zhang B, Lu Y, Yang Y, Ban D, et al. Single nucleotide polymorphism scanning and expression of the pig PPARGC1A gene in different breeds. Lipids. 2014;49:1047-55.

38. Lee JS, Kim JM, Hong JS, Lim KS, Hong KC, Lee YS. Effects of polymorphisms in the $3^{\prime}$ untranslated region of the porcine PPARGC1A gene on muscle fiber characteristics and meat quality traits. Mol Biol Rep. 2012;39:3943-50.

39. Knowles DA, Davis JR, Edgington H, Raj A, Fave MJ, Zhu X, et al. Allelespecific expression reveals interactions between genetic variation and environment. Nat Methods. 2017:14:699-702.

40. Locke JM, Hysenaj G, Wood AR, Weedon MN, Harries LW. Targeted allelic expression profiling in human islets identifies cis-regulatory effects for multiple variants identified by type 2 diabetes genome-wide association studies. Diabetes. 2015;64:1484-91.

41. Milani L, Lundmark A, Nordlund J, Kiialainen A, Flaegstad T, Jonmundsson $G$, et al. Allele-specific gene expression patterns in primary leukemic cells reveal regulation of gene expression by CpG site methylation. Genome Res. 2009;19:1-11.

42. Jones PA. Functions of DNA methylation: islands, start sites, gene bodies and beyond. Nat Rev Genet. 2012;13:484-92.

43. Clarke-Harris R, Wilkin TJ, Hosking J, Pinkney J, Jeffery AN, Metcalf BS, et al. PGC1alpha promoter methylation in blood at 5-7 years predicts adiposity from 9 to 14 years (EarlyBird 50). Diabetes. 2014;63:2528-37.

44. Stachowiak M, Nowacka-Woszuk J, Szydlowski M, Switonski M. The ACACA and SREBF1 genes are promising markers for pig carcass and performance traits, but not for fatty acid content in the longissimus dorsi muscle and adipose tissue. Meat Sci. 2013;95:64-71.
45. Murani E, Ponsuksili S, Reyer H, Wittenburg D, Wimmers K. Expression variation of the porcine ADRB2 has a complex genetic background. Mol Gen Genomics. 2013;288:615-25.

46. Olbromski R, Siadkowska E, Zelazowska B, Zwierzchowski L. Allelic gene expression imbalance of bovine IGF2, LEP and CCL2 genes in liver, kidney and pituitary. Mol Biol Rep. 2013;40:1189-200.
Ready to submit your research? Choose BMC and benefit from:

- fast, convenient online submission

- thorough peer review by experienced researchers in your field

- rapid publication on acceptance

- support for research data, including large and complex data types

- gold Open Access which fosters wider collaboration and increased citations

- maximum visibility for your research: over $100 \mathrm{M}$ website views per year

At $\mathrm{BMC}$, research is always in progress.

Learn more biomedcentral.com/submissions 